\title{
Protocol for Birmingham Atrial Fibrillation Treatment of the Aged study (BAFTA): a randomised controlled trial of warfarin versus aspirin for stroke prevention in the management of atrial fibrillation in an elderly primary care population [ISRCTN89345269]
}

\author{
Jonathan WF Mant*1, Suzanne H Richards1, FD Richard Hobbs"1, \\ David Fitzmaurice ${ }^{1}$, Gregory YH Lip ${ }^{2}$, Ellen Murray ${ }^{1}$, Miriam Banting1, \\ Kate Fletcher ${ }^{1}$, Joy Rahman ${ }^{1}$, Teresa Allan ${ }^{1}$, James Raftery ${ }^{3}$, Stirling Bryan ${ }^{3}$ \\ and the Midlands Research Consortium of General Practice ${ }^{1}$
}

\author{
Address: ${ }^{1}$ Department of Primary Care \& General Practice, University of Birmingham, UK, ${ }^{2}$ University Department of Medicine, City Hospital \\ Birmingham, UK and ${ }^{3}$ Health Service Management Centre, University of Birmingham, UK \\ Email: Jonathan WF Mant* - j.w.mant@bham.ac.uk; Suzanne H Richards - suzanne.richards@pms.ac.uk; FD \\ Richard Hobbs - f.d.r.hobbs@bham.ac.uk; David Fitzmaurice - d.a.fitzmaurice@bham.ac.uk; Gregory YH Lip - g.y.lip@bham.ac.uk; \\ Ellen Murray - e.t.murray@bham.ac.uk; Miriam Banting - m.v.banting@bham.ac.uk; Kate Fletcher - k.fletcher@bham.ac.uk; \\ Joy Rahman - j.rahman@bham.ac.uk; Teresa Allan - t.f.allan@city.ac.uk; James Raftery - j.p.raftery@bham.ac.uk; \\ Stirling Bryan - s.bryan@bham.ac.uk; the Midlands Research Consortium of General Practice - \\ * Corresponding author
}

Published: 26 August 2003

BMC Cardiovascular Disorders 2003, 3:9

Received: 10 June 2003

Accepted: 26 August 2003

This article is available from: http://www.biomedcentral.com//47I-226I/3/9

(C) 2003 Mant et al; licensee BioMed Central Ltd. This is an Open Access article: verbatim copying and redistribution of this article are permitted in all media for any purpose, provided this notice is preserved along with the article's original URL.

\begin{abstract}
Background: Atrial fibrillation (AF) is an important independent risk factor for stroke. Randomised controlled trials have shown that this risk can be reduced substantially by treatment with warfarin or more modestly by treatment with aspirin. Existing trial data for the effectiveness of warfarin are drawn largely from studies in selected secondary care populations that underrepresent the elderly.

The Birmingham Atrial Fibrillation Treatment of the Aged (BAFTA) study will provide evidence of the risks and benefits of warfarin versus aspirin for the prevention of stroke for older people with $\mathrm{AF}$ in a primary care setting.
\end{abstract}

Study design: A randomised controlled trial where older patients with $\mathrm{AF}$ are randomised to receive adjusted dose warfarin or aspirin. Patients will be followed up at three months postrandomisation, then at six monthly intervals there after for an average of three years by their general practitioner. Patients will also receive an annual health questionnaire.

1240 patients will be recruited from over 200 practices in England. Patients must be aged 75 years or over and have AF. Patients will be excluded if they have a history of any of the following conditions: rheumatic heart disease; major non-traumatic haemorrhage; intra-cranial haemorrhage; oesophageal varices; active endoscopically proven peptic ulcer disease; allergic hypersensitivity to warfarin or aspirin; or terminal illness. Patients will also be excluded if the GP considers that there are clinical reasons to treat a patient with warfarin in preference to aspirin (or vice versa).

The primary end-point is fatal or non-fatal disabling stroke (ischaemic or haemorrhagic) or significant arterial embolism. Secondary outcomes include major extra-cranial haemorrhage, death (all cause, vascular), hospital admissions (all cause, vascular), cognition, quality of life, disability and compliance with study medication. 


\section{Background}

Atrial fibrillation (AF) is associated with a $50 \%$ to $90 \%$ increase in all cause mortality, principally due to excess risk of death from cardiovascular diseases including stroke $[1,2]$. It is a particularly important risk factor for stroke in the elderly, since both the incidence of stroke and the prevalence of AF rise with age $[3,4]$. Whilst AF is associated with $15 \%$ of all strokes, it is associated with $36 \%$ of strokes in people over the age of 80 [5]. It is important, therefore, to identify the most effective strategy for stroke prevention for older people with AF.

Four major strategies have been tested in clinical trials to reduce stroke risk in AF including the use of: adjusteddose warfarin; anti-platelet agents such as aspirin; fixed low dose warfarin and fixed low dose warfarin in combination with aspirin. Previous studies have shown that both fixed low dose warfarin and the combination of low dose warfarin with aspirin are significantly less effective than adjusted dose warfarin. [6-9] The principal alternatives to reduce stroke risk in patients with $\mathrm{AF}$ are therefore adjusted dose warfarin or aspirin.

There is strong evidence that adjusted dose warfarin is highly effective in reducing risk of stroke and thromboembolism in people with non-rheumatic AF [10-12]. A meta-analysis of five trials of warfarin therapy suggested that this approach results in a $68 \%$ reduction in risk of stroke [10]. An important side effect of warfarin is haemorrhage, but under trial conditions, observed rates of serious haemorrhage have been low [10]. Aspirin appears to be a safer, but less effective alternative to warfarin resulting in a $22 \%$ reduction in risk of stroke $[12,13]$.

A summary of the seven trials which have directly compared antiplatelet agents to warfarin for stroke prevention in $\mathrm{AF}$ in the relevant age group (where possible) is shown in table 1 . While three studies $[6,14,15]$ found significant benefits of adjusted dose warfarin over aspirin of the order of magnitude that would be anticipated from the indirect comparisons, these findings were not replicated in the other four studies. Indeed, two of the studies showed nonsignificant results in favour of aspirin $[8,16]$. Published meta-analyses of the trials have reached different conclusions due, in part, to controversy [17] as to which trials should be included in this review [12,18]. Notwithstanding this issue, study populations of trials examining the use of warfarin for patients in AF significantly under-represented elderly people. In excess of $50 \%$ of people in $\mathrm{AF}$ in the community would be expected to be over the age of $75,[4]$ whilst only $20 \%$ of patients in the trials were in this age group [10]. In the EAFT study, age was the main reason patients were considered ineligible for the study, accounting for $55 \%$ of exclusions [14]. As a result, the evidence for the effectiveness of warfarin in $\mathrm{AF}$ for patients over the age of 75 is much less impressive than the evidence for patients under this age [19]. Sub-group analysis of a review of five trials suggested that warfarin did reduce stroke risk in this age group, but it was based on only 24 events [10].

There are also concerns over the safety, in terms of increased haemorrhagic risk, of warfarin [18] and this may be particularly important for older age groups. In the SPAF II study, the annual risk of stroke with residual deficit (haemorrhagic and ischaemic) was in fact slightly higher $(4.6 \%$ vs $4.3 \%)$ in patients aged over 75 assigned to warfarin as opposed to aspirin [16]. Factors that might increase risk of major haemorrhage in very elderly patients on warfarin include: deficits in auditory and visual acuity; risk of falls; impaired cognition and memory; dietary vitamin K deficiency; polypharmacy; amyloid angiopathy; and occult gastro-intestinal lesions [19]. A secondary analysis of the SPAF trial data, comparing adjusted dose warfarin versus aspirin, found age to be an important risk factor for major haemorrhage on warfarin [20]. This association of age with risk of haemorrhage has been found in some observational studies, $[21,22]$ but not others [23].

Finally, it is important that a trial performed to investigate the role of anticoagulation in the elderly is carried out in a primary care setting [24]. The existing evidence base for the benefits of anticoagulation is comprised of trials that were mostly conducted on hospital populations. General practitioners (GPs) have questioned the applicability of the results of these studies to primary care settings [25]. Patients were mostly recruited from hospital, and 3\% to $40 \%$ of patients identified with $\mathrm{AF}$ were entered into the trials. While many exclusions were legitimate (e.g. prior indication for warfarin, or clear contra-indication), some were less justifiable (for example about a third of patients in the SPAF II study were excluded for reasons that may have related to perceived haemorrhage risk). It is noteworthy that concerns over risk of haemorrhage were found to be the main barrier to anticoagulant use by primary care physicians in the USA [26]. If GPs assume that the risks of haemorrhage in their practice are higher than was observed in the trials, the perceived harm/benefit ratio for individual patients is changed. Therefore, to improve the likelihood that results of the proposed study are acted upon, it is important that it is carried out in a setting which is representative of primary care [24].

Overall, the limitations of the evidence base for management of AF in elderly primary care population is reflected in low uptake of anticoagulation in clinical practice. Audits of use of warfarin in AF have consistently demonstrated low uptake of the therapy, particularly in the elderly. Recent studies based in defined UK general practice 
Table I: Randomised controlled trials which have compared aspirin with adjusted dose warfarin in the treatment of atrial fibrillation

\begin{tabular}{|c|c|c|c|c|c|}
\hline Study & Population & Age & Interventions & Primary end-point & $\begin{array}{l}\text { Relative risk of } \\
\text { primary end-point } \\
(95 \% \mathrm{Cl})\end{array}$ \\
\hline $\begin{array}{l}\text { AFASAK I } \\
{[15] .}\end{array}$ & $\begin{array}{l}\text { Primary care. } N=67 \mathrm{I} \\
\text { (in relevant arms) }\end{array}$ & $\begin{array}{l}\text { No age limits. } \\
\text { Mean age } 74 \text { yrs. }\end{array}$ & $\begin{array}{l}3 \text { arm study including } \\
\text { warfarin (INR 2.8-4.2) and } \\
\text { aspirin (75 mg). }\end{array}$ & $\begin{array}{l}\text { Stroke, transient ischaemic } \\
\text { attack and systemic } \\
\text { embolus. }\end{array}$ & $\begin{array}{l}\text { Not given. RR on } \\
\text { warfarin as compared } \\
\text { to aspirin or placebo: } \\
0.36\end{array}$ \\
\hline EAFT [14]. & $\begin{array}{l}\text { TIA or minor stroke } \\
\text { in preceding } 3 / 12 \mathrm{~N}= \\
455 \text { (in relevant arms) }\end{array}$ & $65 \%$ were $>69$ yrs & $\begin{array}{l}3 \text { arm study, including } \\
\text { anti-coagulant (INR } 2.5- \\
4.0 \text { ) and aspirin ( } 300 \mathrm{mg})\end{array}$ & $\begin{array}{l}\text { Death from vascular } \\
\text { disease; non-fatal stroke } \\
\text { (including haemorrhage); } \\
\text { non-fatal myocardial } \\
\text { infarction or systemic } \\
\text { embolus }\end{array}$ & $0.60(0.4 \mid$ to 0.87$)$ \\
\hline SPAFII[16]. & $N=385$ & $>75$ yrs & $\begin{array}{l}\text { Warfarin (INR 2.0-4.5) vs } \\
\text { aspirin ( } 325 \mathrm{mg}) \text {. }\end{array}$ & $\begin{array}{l}\text { Ischaemic stroke and } \\
\text { systemic embolus }\end{array}$ & $\begin{array}{l}0.73 \text { ( } 0.37 \text { to I.5) } 1.07 \\
\text { (stroke with residual } \\
\text { deficit including } \\
\text { haemorrhagic) }\end{array}$ \\
\hline AFASAKII[8]. & $\begin{array}{l}\text { Primary care. } N=339 \\
\text { (in relevant arms) }\end{array}$ & $\begin{array}{l}\text { No upper age } \\
\text { limit. Mean age } 73 \\
\text { yrs. }\end{array}$ & $\begin{array}{l}3 \text { arm study, including } \\
\text { warfarin (INR 2.0-3.0) and } \\
\text { aspirin }(300 \mathrm{mg}) \text { arms. }\end{array}$ & $\begin{array}{l}\text { Stroke (ischaemic or } \\
\text { haemorrhagic) or systemic } \\
\text { thrombo-embolus. }\end{array}$ & $\begin{array}{l}1.26 \text { (intention to } \\
\text { treat) } 0.78 \text { (treatment } \\
\text { received analysis) }\end{array}$ \\
\hline SIFA [43]. & $\begin{array}{l}\text { Recent cerebral } \\
\text { ischaemia } N=916\end{array}$ & $\begin{array}{l}\text { Age }>30 \text { yrs Mean } \\
\text { age } 72 \text { yrs. }\end{array}$ & $\begin{array}{l}\text { Warfarin (INR } 2.0-3.5) \text { vs } \\
\text { indobufen ( } 200 \mathrm{mg} \text { bd or } \\
100 \mathrm{mg} \text { bd if creatinine } \\
\text { clearance }<80 \mathrm{mls} / \mathrm{min})\end{array}$ & $\begin{array}{l}\text { Stroke (including } \\
\text { haemorrhagic), myocardial } \\
\text { infarction, pulmonary } \\
\text { embolus, systemic embolus } \\
\text { or vascular death }\end{array}$ & 0.85 (not significant) \\
\hline SPAFIII [6]. & $\begin{array}{l}\text { At least I risk factor } \\
\text { for stroke } N=1044\end{array}$ & $\begin{array}{l}\text { No upper age } \\
\text { limit. Mean age 7I } \\
\text { yrs. }\end{array}$ & $\begin{array}{l}\text { Warfarin (INR 2.0-3.0) vs } \\
\text { aspirin ( } 325 \mathrm{mg} \text { ) and fixed } \\
\text { mini-dose warfarin (INR } \\
\text { I.2-I.5). }\end{array}$ & $\begin{array}{l}\text { Ischaemic stroke and } \\
\text { systemic embolus }\end{array}$ & $0.26(0.13$ to 0.50$)$ \\
\hline PATAF [9]. & $\begin{array}{l}\text { Primary care. } N=272 \\
\text { (in relevant arms) }\end{array}$ & $\begin{array}{l}\text { Age } 60-78 . \text { Mean } \\
\text { age } 70 \text { yrs. }\end{array}$ & $\begin{array}{l}3 \text { arm study including } \\
\text { warfarin (INR 2.5-3.5) and } \\
\text { aspirin (I50 mg). }\end{array}$ & $\begin{array}{l}\text { Stroke, systemic embolus, } \\
\text { major haemorrhage or } \\
\text { vascular death. }\end{array}$ & 0.78 (0.34 tol. 8$)$ \\
\hline
\end{tabular}

[In the relative risk column, the risk of an end-point on variable dose warfarin is compared to the risk on aspirin (and fixed-minidose warfarin in the case of SPAF-III). Thus, a relative risk greater than one favours aspirin, and a relative risk less than one favours warfarin]

populations have found that only between $18 \%$ and $40 \%$ of people in AF without contra-indications for therapy were being treated with warfarin. [27-29] Sudlow et al found that only $17 \%$ of patients in AF over the age of 74 were on warfarin [30]. There is also no clinical consensus with regard to use of anticoagulation in the elderly. In a review of the content of guidelines on anticoagulant treatment in AF in Great Britain, Thomson et al found that between $13 \%$ and $100 \%$ of patients with AF would be treated depending upon which guideline was used [31]. An important cause of this variation was different advice with regard to age. Ten of 20 guidelines referred to the higher risks of anticoagulation in the elderly, and eight gave upper age limits, with four recommending not treating people over the age of 80 , and two suggesting that anticoagulation should be used in people over the age of 75 only in exceptional circumstances. If, as is current practice, the majority of patients over the age of 75 with AF are not treated with warfarin, targeting this group of patients is likely to have a substantial public health benefit in terms of preventing stroke [32] if warfarin is indeed more effective than aspirin.

In conclusion, although there is a good evidence basis to support adjusted-dose warfarin as the most effective strategy for the prevention of stroke in $\mathrm{AF}$, there are limitations when applying these data to older people (75 years or over) and a trial focusing on this age group is needed [19]. The Birmingham Atrial Fibrillation Treatment of the Aged Study (BAFTA) aims to provide robust data on the effectiveness, in terms of stroke prevention and haemorrhagic risk, of adjusted dose warfarin versus aspirin in older patients with AF identified from primary care settings.

\section{Methods \\ Study Aims}

The primary aim of BAFTA is to compare the incidence of fatal and non-fatal disabling stroke (ischaemic or haemorrhagic) and systemic embolism in older patients with $\mathrm{AF}$ who are treated with either adjusted dose warfarin or aspirin. 


\section{Study design and setting}

BAFTA is a primary-care based, pragmatic randomised controlled trial where older people (aged 75 years or over) with $\mathrm{AF}$ are randomised to receive either warfarin or aspirin. The pathway by which patients are recruited is given in figure 1. A full economic evaluation is also being conducted.

\section{Ethical considerations}

Full ethical approval for this study has been obtained. Two external bodies, a Data Monitoring and Ethics Committee and a Trial Steering Committee, will monitor study progress.

\section{Study interventions}

Adjusted dose warfarin with a target international normalised ratio (INR) of 2.5 (acceptable range of 2.0 to 3.0) was selected as this is the recommended strategy of the British Society of Haematology for the treatment of AF [33]. Warfarin dosage and INR monitoring may be carried out in primary care or hospital clinics, depending on whichever is the standard procedure for the participating practice. Methods for adjusting warfarin dose and monitoring INR are at the discretion of the local practices and hospital anticoagulation clinics. Patients allocated aspirin will receive $75 \mathrm{mg}$ daily as this dose has been shown to be as effective as higher doses (e.g. $300 \mathrm{mg}$ ) in the prevention of stroke [34].

\section{Identification of eligible patients}

Eligible patients will be identified from general practices in England selected mainly from the West Midlands. Larger practices (three or more partners) will be recruited in preference to smaller ones. Each practice will receive a start-up visit by a member of the research team who will run a practice-based computer search to identify all patients aged 74 years or over. Within this population, patients with probable AF will be identified in three ways: by computerised searches of GP records; by opportunistic screening of the pulse by practice staff and by considering patients who present with previously undiagnosed AF ('incident cases').

The computerised note searches of general practice records will be tailored towards the information held in each practice and will identify patients who have already been diagnosed as having AF. Patients in this group will be invited to attend an ECG clinic at their practice. The opportunistic screening will take place by placing a flag (paper or computerised) in the case notes of all patients aged 74 years or over who were not identified as being in $\mathrm{AF}$ as a result of the computer searching. The flag prompts a member of the primary health care team to take the patient's pulse the next time the patient consults. It is anticipated that over 12 months, $91 \%$ of patients in this age range will have been seen at least once by their GP [35]. Pulse palpation is a highly sensitive (though nonspecific) way of detecting AF (sensitivity = 94\%) [36]. The result of the pulse check (regular or irregular) is recorded and those patients with an irregular pulse will be invited to an ECG clinic.

\section{Confirmation of diagnosis of AF: the ECG clinic}

The practice nurse records a 12 lead ECG and collects other baseline information (table 3 ) at a dedicated clinic. The ECG is sent to the study team to be read by a cardiologist, who will report whether AF is present or absent and list any other significant abnormalities. Where the ECG shows sinus rhythm, but the patient has a case note diagnosis of AF, records of ECGs and relevant case notes over the previous two years will be reviewed. If there is evidence of chronic atrial fibrillation (permanent or paroxys$\mathrm{mal}$ ) in the case records, including evidence of at least one ECG in the last two years showing AF, then this patient will be considered eligible. Cases where the AF appears transient or self-limiting are excluded. Patients identified as an incident case, who had an ECG outside the BAFTA study, will have this ECG submitted to the BAFTA cardiologists for reading and do not need to be called to the dedicated nurse clinic.

\section{Determining eligibility for the study}

Eligibility for the study is determined in two stages. Firstly, the practice nurse, in consultation with the GP, reviews the patient's medical records to check whether the patient satisfies the study inclusion criteria and does not have any of the absolute exclusion criteria listed in table 2. Secondly, the GP decides whether there is clinical uncertainty as to whether the patient should be treated with aspirin or warfarin, taking into account other clinical factors (see table 2, and randomisation clinic below). Eligible patients are invited to a randomisation clinic appointment. All patients will have received a detailed information sheet about the BAFTA study before their randomisation clinic appointment. Patients who are not eligible for a randomisation clinic appointment are followed-up at the GP's discretion.

\section{Randomisation clinic}

At the randomisation clinic the GP once again checks to ensure that the patient satisfies both inclusion and absolute exclusion criteria. Patients with temporary exclusion criteria (table 2) are not eligible for the study, though may be re-considered at a later date.

The decision as to whether or not the patient is eligible for trial entry is made by the GP using the uncertainty principle. That is, if a GP is certain that a patient should not be entered into the trial, for whatever reason, that patient is not eligible for entry into BAFTA. If the GP is substantially 


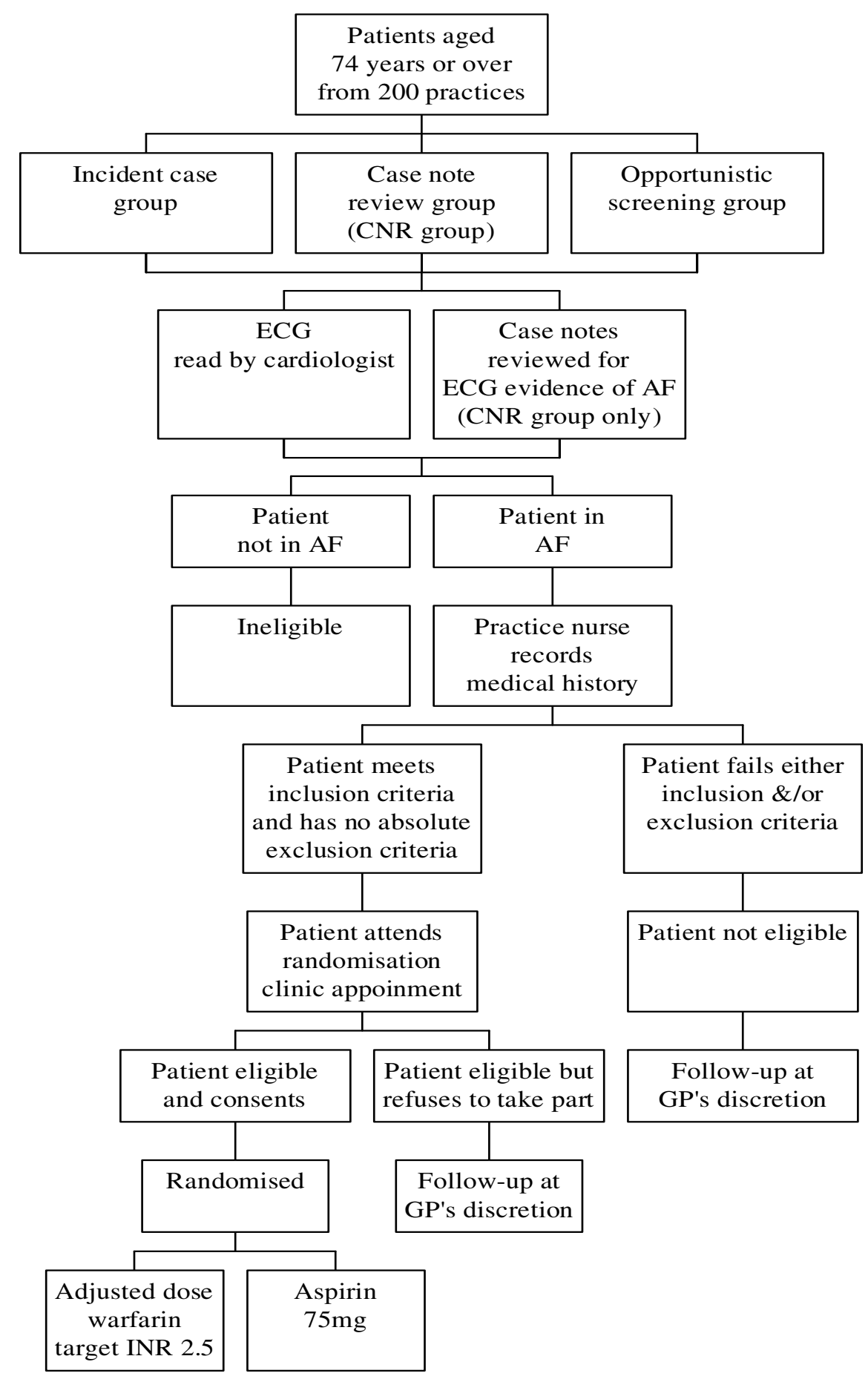

Figure I

Summary of trial design. Incident case group: patient presents with atrial fibrillation to GP; Case note review group: patient is identified through GP computer system; Opportunistic screening group: patient is identified through opportunistic taking of pulse. 
Table 2: Study inclusion and exclusion criteria

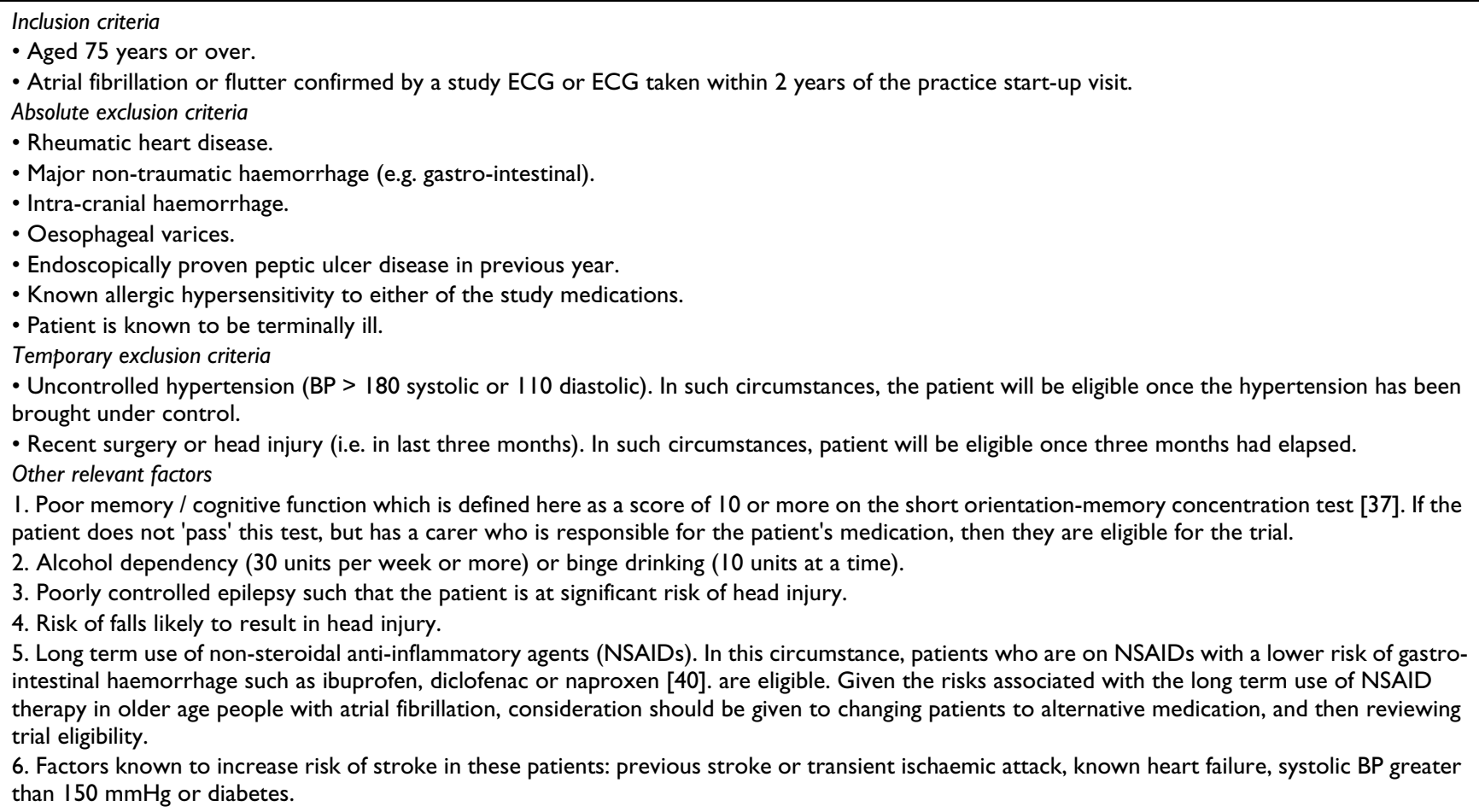

uncertain whether or not warfarin is indicated, then the patient is eligible for BAFTA. This decision will need to be made on a case by case basis after consideration of all relevant factors listed in table 2. Patients already on warfarin or aspirin are eligible for consideration. In each case, factors which increase risk of haemorrhage (points 1 to 5) need to be set against the high risk of stroke in these patients, which is increased still further by certain factors (point 6). If the GP remains uncertain as to whether or not the patient should be on warfarin or aspirin, the patient will be offered trial entry. Patients with substantial cognitive impairments (defined as 10 or more on the Short Orientation Memory Concentration test [37] or 89 at the GP's discretion) can be considered for trial entry as long as a carer from the patient's family is willing to support the patient's entry into the trial, and the GP is satisfied that warfarin could be safely administered.

If the patient (and carer where appropriate) is willing to take part, the GP then obtains written consent from the patient (and carer) prior to randomisation. The randomisation will be stratified by age into three strata (75-79; 80-84; and 85+) with gender balanced by minimisation. The randomisation schedule has been developed by an independent trials unit, and the random allocation is obtained by GPs telephoning this randomisation service. Once the treatment allocation has been obtained, the GP then prescribes the trial medication and arranges the appropriate clinical follow up. Patients who are either ineligible for trial entry or who do not wish to take part in the study are treated at the discretion of the GP.

\section{Patient follow-up procedures}

Patients will be followed up from trial entry until the end of the study (anticipated average: three years). Follow-up is composed of several elements. First, at three months post-randomisation and then six monthly intervals there afterwards, the GP will review each patient and record evidence of any primary or secondary end-points (listed in table 3 ) and compliance with trial medication. Second, at six monthly intervals but three months after the GP review, a researcher will review the patient's medical records and note whether or not primary and secondary outcomes have occurred. Third, at twelve months postrandomisation and annually there afterwards the patient will be sent a postal health questionnaire (described in table 3). Non-responders will be sent a second questionnaire within three weeks, and then contacted by telephone if they fail to respond after two successive mailings. The records of patients will be flagged at the NHS Central Register to ensure that the study team is informed about any deaths that occur. Finally, INR data for patients on warfarin will be obtained from their hospital or GP records. 
Table 3: Timing and content of study assessments

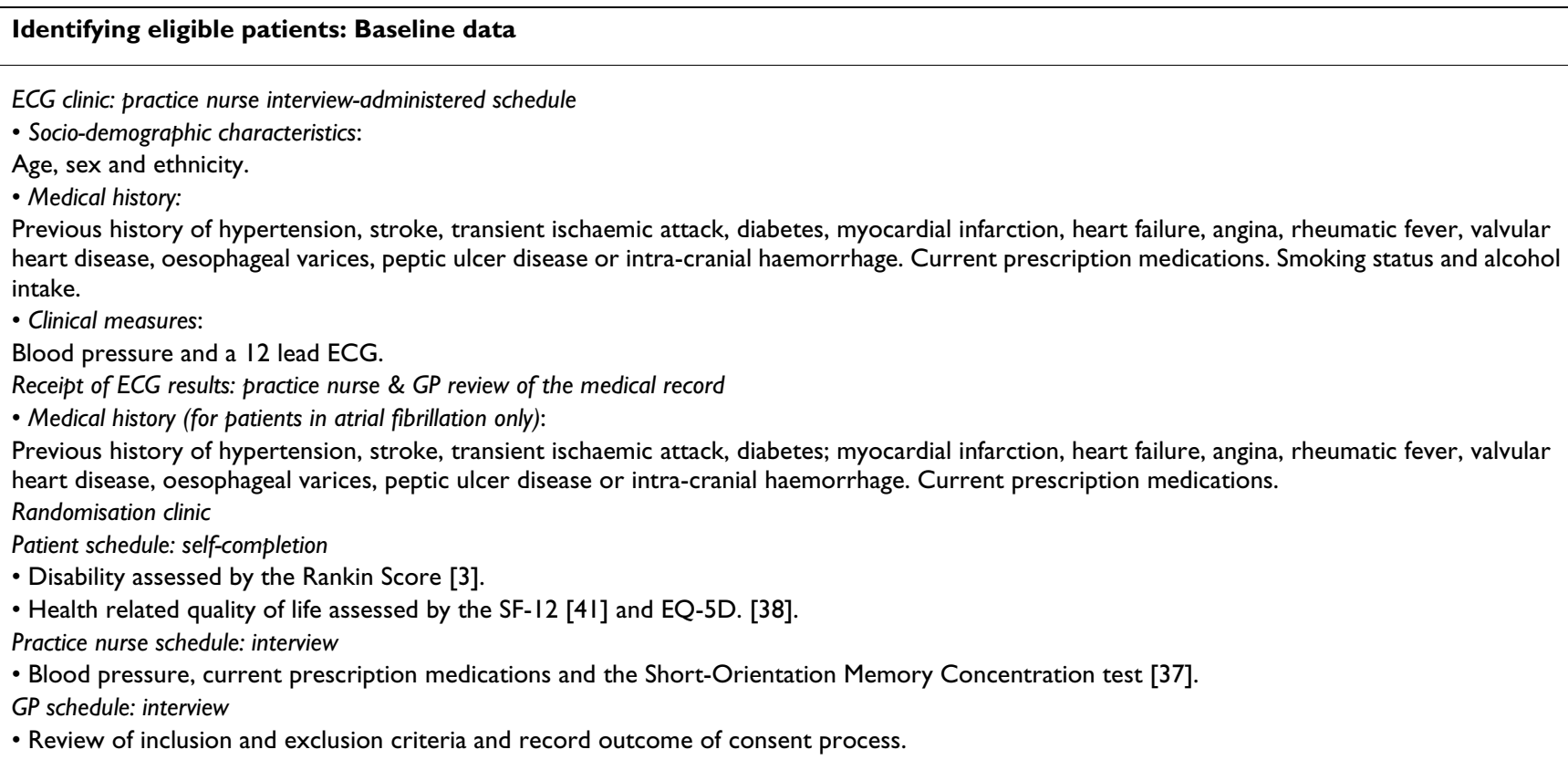

\section{Patient follow-up procedures: for an average of three years}

Obtaining information on patients who died

- Records flagged at NHS central register.

GP interviews at 3 months post-randomisation, then six monthly intervals there afterward

- Major extra-cranial haemorrhage (fatal, or one that requires transfusion or surgery).

- Death - all cause, all vascular and stroke.

- Admission to hospital - all cause, all vascular, stroke.

- Cognition assessed by Mini-Mental State Exam [42]. at (9, 21, and 33 month follow-ups only).

- Disability assessed by the patient using the Rankin Score [3].

- Blood pressure and apical pulse rate.

- Drop out/withdrawal from allocated medication.

Researcher case note review at 6 months post-randomisation, then six monthly intervals there afterward

- Major extra-cranial haemorrhage (fatal, or one that requires transfusion or surgery).

- Death - all cause, all vascular and stroke.

- Admission to hospital - all cause, all vascular, stroke.

- Drop out/withdrawal from allocated medication.

Patient self-completed postal questionnaire at 12 months post-randomisation, then annually there afterwards

- Disability assessed by the Rankin Score [3].

- Health related quality of life assessed by the SF-12 [4I] and EQ-5D [38].

- Patient costs questionnaire (warfarin patients only, at 12 months).

\section{Patient outcome measures}

The criteria used to assess whether or not the patient is suitable for inclusion in the study, and the primary and secondary outcome measures assessed across the followup period for patients randomised are summarised in table 3.

The primary outcome is the incidence of fatal or non-fatal disabling stroke (ischaemic or haemorrhagic), intra-cranial haemorrhage or significant arterial embolism. A disa- bling stroke is defined as a Rankin Score [3] of 2 to 5 one month or more after stroke, or a deterioration in the score if the baseline Rankin Score was greater than or equal to 2. If the stroke led to a hospital admission of 30 days or more, this is also classified as a disabling stroke. Intra-cranial haemorrhage will require verification through brain imaging and includes sub-dural haemorrhage and subarachnoid haemorrhage. Significant arterial embolism also requires verification by vascular imaging, scintigra- 
phy, surgery or autopsy. Pulmonary embolism is not included as a primary outcome.

When a possible primary end-point is identified in the study population, the hospital discharge summary, results of brain imaging and a copy of the death certificate (if applicable) will be obtained. These data will be scrutinised by two neurologists independently, blind to the treatment allocation. They will decide whether or not a primary end-point has occurred and classify strokes as thrombo-embolic, haemorrhagic, or uncertain.

A variety of secondary outcome measures are assessed during the GP follow-ups, researcher case note review and the annual patient questionnaire (table 3). Key secondary outcomes include: major extra-cranial haemorrhage (defined as a fatal haemorrhage, or one that requires transfusion or surgery); all stroke (including non-disabling stroke); vascular deaths; all deaths; and vascular admissions (including myocardial infarction and pulmonary embolus). Since the GPs providing clinical care are unblinded, pulse and blood pressure are being monitored to reflect any possible differential treatment in the two groups (for example, better treatment of hypertension or heart failure). Key secondary events (deaths, extracranial major haemorrhage; vascular admissions) will be reviewed by an independent clinician blinded to treatment allocation to ensure unbiased coding of these events.

\section{Sample size considerations}

Randomisation of 1240 patients (620 in each arm), with an average of three years follow-up, will detect a 33\% reduction in the relative risk of acute stroke (ischaemic or haemorrhagic) or arterial embolus between groups with $90 \%$ power $($ alpha $=0.05)$. This figure assumes that the annual incidence of the primary end-point on aspirin is $9 \%,[6,16]$ the annual rate of primary end-points or death will be $14 \%$ and that loss to follow up will be $1 \%$.

To recruit 1240 patients from primary care, an estimate of the number of practices is needed. It was assumed that 7\% of the general population are aged 75 years or over and about $9 \%$ of this age range will be in AF [4]. Of these, 35\% might be expected to have a contra-indication to warfarin [27] and $17 \%$ have an indication for warfarin [30]. This suggests that $48 \%$ of patients identified as being in $\mathrm{AF}$ will be eligible to enter the trial. If $75 \%$ of eligible patients are identified, and $50 \%$ of these agree to enter the trial, this would result in a recruitment rate of 113 per 100,000 practice population. Therefore, to recruit 1240 patients a total practice population of $1,097,000$ is required comprising 76,800 aged 75 years or over. This equates to 157 practices with an average list size of 7,000 and recruitment of eight patients per practice.

\section{Statistical analysis}

The primary analysis will be a comparison of warfarin with aspirin for prevention of the primary end-point following the "intention to treat" principle. Intention to treat analyses will also be performed for the secondary outcomes. Analyses of primary and secondary endpoints, comparing time to event in the two arms, will be performed using the log rank method. Cox regression will also be carried out. All significance tests will be two-sided. Pre-planned sub-group analysis will be performed for the following sub-groups: age (75-79, 80-84, 85+ years) and gender; method of identification of AF (opportunistic screening/computer search identified); history of prior stroke or transient ischaemic attack (yes/no); on warfarin prior to study entry (yes/no); and study ECG showing atrial fibrillation or sinus rhythm (as pragmatic distinction between permanent and paroxysmal atrial fibrillation). A secondary on-treatment analysis will be performed to explore the risks of major haemorrhage. We will also explore the possible effects of other patient characteristics such as blood pressure and concomitant use of other nonsteroidals.

\section{Economic evaluation}

The economic evaluation will adopt a broad perspective, including costs incurred within the health sector and by patients and carers. Data collection will be undertaken on all trial patients in order to allow a stochastic cost analysis to be conducted. The focus of the data collection will be upon the key cost drivers which will include: drug and INR monitoring, contacts with secondary care; primary care visits. The analysis will adopt an incremental approach such that data collection will concentrate on resource use differences between trial arms. The process of collecting data on resource use will be undertaken separately from data collection on unit costs. Resource use data will principally be collected within the trial. Unit costs will be collected from published sources and a representative sample of NHS providers in order to increase generalisability. The methods to be used in collecting data will include a review of patient records (both GP and hospital), whilst private costs data will be collected from a survey of a sub-cohort of the trial population.

\section{Plan of analysis}

It is not possible to state with certainty at the outset which form of economic analysis will be employed, since this will be driven in part by the trial results. The plan for the analysis is to report a cost consequence analysis, which will involve providing a full description of all important results relating to costs and consequences. If no differences in consequences are observed, then a cost minimisation analysis will be conducted. If differences in consequences are observed, then a cost utility analysis will be conducted using data on EQ-5D [38] to estimate Qual- 
ity Adjusted Life Years. Whichever form of analysis is employed, an incremental approach will be used. To predict overall programme costs, the mean costs per patient will be compared using either parametric or non-parametric methods depending on the distribution of the data [39].

Longer term costs and consequences will be explored by extrapolating beyond the end of the trial using a modelling framework. The precise form of modelling is yet to be determined, but is likely to be either Markov or Discrete Event Simulation, depending upon the extent to which the Markov assumptions are justified. An advantage of using such an approach is that it will allow the additional costs of increasing survival to be explicitly incorporated into the analysis.

The robustness of the results of the economic analysis will be explored using sensitivity analysis. This will explore uncertainties in the trial based data itself, the methods employed to analyse the data and the generalisability of the results to other settings. Uncertainty in the confidence to be placed on the results of the economic analysis will be explored by estimating cost-effectiveness acceptability curves. These plot the probability that the intervention is cost-effective against threshold values for cost-effectiveness.

\section{Time plan for the BAFTA study}

Patient recruitment began in April 2001 and is planned to continue until December 2004. By August 2003, 382 patients ( $31 \%$ of target) have been recruited into the trial.

\section{Competing interests}

None of the authors have any competing interests arising from this research.

\section{Contributions of authors}

Jonathan Mant, Richard Hobbs, David Fitzmaurice, Gregory YH Lip, Ellen Murray, Teresa Allan, Stirling Bryan and James Raftery were responsible for identifying the research question, and contributing to drafting of the study protocol. Suzanne Richards, Miriam Banting, Kate Fletcher and Joy Rahman have all contributed to the development of the protocol and study design, as members of the research team. Jonathan Mant and Suzanne Richards were responsible for the drafting of this paper, although all authors provided comments on the drafts and have read and approved the final version.

\section{Acknowledgements}

The BAFTA research team would like to thank Victoria Hurley for her contribution to the research team. We would also like to thank the Trial Steering Committee members of Dr Colin Baigent, Reverend Dr Barry Clark, Professor Stuart Cobbe (Chairman), Professor Godfrey Fowler, Professor Tom Meade, Dr Mark Sudlow and Professor Keith Wheatley for their inval- uable comments that have much improved the BAFTA protocol. Similarly, we would also like to acknowledge the input from the Data Monitoring and Ethics Committee members of Professor Robert Hart, Professor Tim Peters and Professor Peter Sandercock (Chairman). Finally we would like to thank all of the general practitioners and practice nurses who are running study clinics, and for feedback on how to translate a study protocol into a model that will work in the challenging environment of primary care.

The study is funded by the Medical Research Council.

We also acknowledge the support provided by the MidReC (Midlands Research Practices Consortium), and by Hertnet (the Hertfordshire network of practices).

\section{References}

I. Benjamin E, Wolf P, D'Agostino R, Silbershatz H, Kannel W and Levy $D$ : Impact of atrial fibrillation on the risk of death: the Framingham Heart Study. Circulation 1998, 98:946-952.

2. Wolf $P$, Abbott $R$ and Kannel $W$ : Atrial fibrillation as andependent risk factor for stroke: The Framingham Study. Stroke |99|, 22:983-988.

3. Bamford J, Sandercock P, Dennis M, Burn J and Warlow C: A prospective study of acute cerebrovascular disease in the community: the Oxfordshire Community Stroke Study - 198 I86. Incidence, case fatality rates and overall outcome at one year of cerebral infarction, primary intracerebral and subarachnoid haemorrhage. J Neurol Neurosurg Psychiatry 1990, 53:16-22.

4. Feinberg W, Blackshear J, Laupacis A, Kronmal R and Hart R: Prevalence, age distribution and gender of patients with atrial fibrillation. Arch Intern Med 1995, I 55:469-473.

5. Wolf $P, A b b o t t R$ and Kannel W: Atrial fibrillation: a major contributor to stroke in the elderly. The Framingham Study. Arch Intern Med 1987, I 47: I56I-I564.

6. Stroke Prevention in Atrial Fibrillation Investigators: Adjusted dose warfarin versus low-intensity, fixed dose warfarin plus aspirin for high-risk patients with atrial fibrillation: stroke prevention in atrial fibrillation III RCT. Lancet 1996, 348:633-638.

7. Pengo V, Zasso A, Barbero F, Banzato A, Nante G, Parissenti L, John $N$, Noventa F and Dalla Volta F: Effectiveness of fixed minidose warfarin in the prevention of thromboembolism and vascular death in nonrheumatic atrial fibrillation. Am J Cardiol I998, 82:433-437.

8. Gullov AL, Koefoed BG, Petersen P, Pedersen TS, Andersen ED, Godtfredsen J and Boysen G: Fixed minidose warfarin and aspirin alone and in combination vs adjusted dose warfarin for stroke prevention in atrial fibrillation. Arch Intern Med 1998, I58:1513-1521.

9. Hellemons BS, Langenberg M, Lodder J, Vermeer F, Schouten HJ, Lemmens T, van Ree JW and Knottnerus JA: Primary prevention of arterial thromboembolism in non-rheumatic atrial fibrillation in primary care: RCT comparing two intensities of coumarin with aspirin. BMJ 1999, 3 19:958-964.

10. Atrial Fibrillation Investigators: Risk factors for stroke and efficacy of antithrombotic therapy in atrial fibrillation. Analysis of pooled data from five randomised controlled trials. Arch Intern Med 1994, I 54:|449-|457.

II. Ezekowitz $M$ and Levine J: Preventing stroke in patients with atrial fibrillation. JAMA 1999, 28I: I I83-I835.

12. Hart R, Benavente O, McBride R and Pearce L: Antithrombotic therapy to prevent stroke in patients with AF: a meta-analysis. Ann Intern Med 1999, I 3 I:492-501.

13. Atrial Fibrillation Investigators: The efficacy of aspirin in patients with atrial fibrillation. Arch Intern Med 1997, I 57: I 237-I240.

14. European Atrial Fibrillation Study group (EAFT): Secondary prevention in non-rheumatic atrial fibrillation after transient ischaemic attack or minor stroke. Lancet 1993, 342: I255-I 262.

15. Petersen P, Boysen G, Godtfredsen J, Andersen E and Andersen B: Placebo-controlled, randomised trial of warfarin and aspirin for prevention of thrombo-embolic complications in chronic atrial fibrillation: the Copenhagen AFASAK study. Lancet I989, I(863I): | 75-| 78 . 
16. Stroke Prevention in Atrial Fibrillation Investigators: Warfarin versus aspirin for prevention of thromboembolism in atrial fibrillation: Stroke Prevention in Atrial Fibrillation II Study. Lancet 1994, 343:687-69|.

17. Mant J, Fitzmaurice D, Murray E, Lip G and Hobbs F: Inclusion criteria determine results of review. BMJ 200I, 323:233-34.

18. Taylor F, Cohen $H$ and Ebrahim S: Systematic review of long term anticoagulation or aniplatelet treatment in patients with non-rheumatic atrial fibrillation. BMJ 200I, 322:321-326.

19. Marine J and Goldhaber S: Controversies surrounding long term anti-coagulation of very elderly patients in atrial fibrillation. Chest 1998, I I3: I I I5- I I I8.

20. Stroke Prevention in Atrial Fibrillation Investigators: Bleeding during antithrombotic therapy in patients with atrial fibrillation. Arch Intern Med 1996, I 56:409-4I6.

2I. Palareti G, Hirsh J, Legnani C, Manotti C, D'Angelo A, Pengo V, Moia M, Guazzaloca G, Musolesi S and Coccheri S: Oral anticoagulation treatment in the elderly: a nested prospective case control study. Arch Intern Med 2000, I60:470-478.

22. Palareti G, Leali N, Coccheri S, Poggi M, Manotti C, D'Angelo A Pengo V, Erba N, Moia M, Ciavarella N, Devoto G, Berrettini M and Musolesi S: Bleeding complications of oral anticoagulant treatment: an inception cohort, prospective collaborative study (ISCOAT). Lancet 1996, 348:423-428.

23. Fihn S, Callahan C, Martin D, McDonnell M, Henikoff J and White R: The risk for and severity of bleeding complications in elderly patients treated with warfarin. Ann Intern Med 1996, I 24:970-979.

24. Medical Research Council: MRC Topic review - Primary Health Care. London, Medical Research Council 1997.

25. Sweeney KG, Gray DP, Steele R and Evans P: Use of warfarin in NRAF: a commentary from general practice. $\mathrm{Br} J$ Gen Prac 1995, 45:153-158.

26. McCrory D, Matchar D, Samsa G, Sanders L and Pritchett E: Physician attitudes about anticoagulation for nonvalvular atrial fibrillation in the elderly. Arch Intern Med 1995, I 55:277-28I.

27. Sudlow M, Rodgers H, Kenny R and Thomson R: Population based study of use of anticoagulants among patients with atrial fibrillation in the community. BMJ I997, 3 | 4: I529- I530.

28. Lip G, Golding D and Nazir M: A survey of atrial fibrillation in general practice: the West Birmingham Atrial Fibrillation Project. Bri J Gen Prac 1997, 47:285-289.

29. Wheeldon $\mathrm{N}$ and Tayler $\mathrm{D}$ : Screening for atrial fibrillation in primary care. Heart 1998, 9:50-55.

30. Sudlow M, Thomson R, Thwaites B, Rodgers H and Kenny R: Prevalence of atrial fibrillation and eligibility for anticoagulants in the community. Lancet 1998, 352:1167-II71.

31. Thomson R, McElroy $\mathrm{H}$ and Sudlow M: Guidelines on anticoagulant treatment in atrial fibrillation in Great Britain: variation in content and implications for treatment. BMJ 1998, 3 1 6:509-5।3.

32. Lancaster $T$, Mant $\mathrm{J}$ and Singer $\mathrm{D}$ : Stoke prevention in atrial fibrillation. BMJ 1997, 3 | 4: | 563-1564.

33. Haemostasis and Thrombosis Task Force: Guidelines on Anticoagulation. Bri J Haematol 31998, 10 1:374-387.

34. Johnson E, Lanes S, Wentworth C, Satterfield M, Abebe B and Dicker $\mathrm{L}$ : A meta-regression analysis of the dose-response effect of aspirin on stroke. Arch Intern Med 1999, I 59:1 248-I253.

35. Office of Population Censuses and Surveys: Morbidity statistics from general practice 199 I/2 (MSGP4). London, HMSO 1994.

36. Sudlow M, Rodgers H, Kenny R and Thomson R: Identification of patients with atrial fibrillation in general practice: a study of screening methods. BMJ 1998, 31 7:327-328.

37. Katzman R, Brown T and Fuld P: Validation of a short orientation memory-concentration test of cognitive impairment. Am J Psychiatry 1983, 40:734-739.

38. The EuroQol Group: EQ-5D user guide. Rotterdam, Netherlands, The EuroQol Group 1996.

39. Thompson $S$ and Barber J: How should cost data in pragmatic trials be analysed? $B M / 2000,320: 1197-2000$

40. Langman MJ, Weil J, Wainwright P, Lawson DH, Rawlins MD, Logan RF, Murphy M, Vessey MP and Colin-Jones DG: Risks of bleeding peptic ulcer associated with individual NSAIDs. Lancet 1994 343:1075-1078.
4I. Ware JE, Kosinski M and Keller S: A I2-item short-form Health survey - construction of scales and preliminary tests of reliability and validity. Med Care 1996, 34:220-233.

42. Folstein MF and Folstein SE: "Mini-Mental State" A practica method for grading the cognitive state of patients for the clinician. J Psychiatric Res 1975, I 2: I89-198.

43. Morocutti C, Amabile G, Fattaposta F, Nicolosi A, Matteoli S, Trappolini M, Cataldo G, Milanesi G, Lavezarri M, Pamparana $F$ and Cocherri S: Indobufen versus warfarin in the secondary prevention of major vascular events in non rheumatic atrial fibrillation. Stroke 1997, 28:1015-1021

\section{Pre-publication history}

The pre-publication history for this paper can be accessed here:

http://www.biomedcentral.com/1471-2261/3/9/prepub
Publish with Bio Med Central and every scientist can read your work free of charge

"BioMed Central will be the most significant development for disseminating the results of biomedical research in our lifetime. "

Sir Paul Nurse, Cancer Research UK

Your research papers will be:

- available free of charge to the entire biomedical community

- peer reviewed and published immediately upon acceptance

- cited in PubMed and archived on PubMed Central

- yours - you keep the copyright
BiolMedcentral 